\title{
Riesgo suicida y desesperanza en pacientes psiquiátricos hospitalizados
}

\author{
Shirley Hawkins ${ }^{\star}$, Alberto Valencia ${ }^{\star *}$, Beatriz Caamaño ${ }^{\star * *}$, Guillermo Ceballos ${ }^{\star * * *}$
}

* Estudiante de Psicología, Universidad del Magdalena, Santa Marta, Colombia.

Correo electrónico:

s.hawkins.m@hotmail.com

** Estudiante de Psicología, Universidad del Magdalena, Santa Marta, Colombia.

Correo electrónico: aba7_1@hotmail.com

*** Médico psiquiatra. Profesor de la Universidad del Magdalena, Santa Marta, Colombia.

Correo electrónico:

tizhelena@gmail.com

**** Especialista en Gerencia de la Calidad en Servicios de la Salud. Profesor de la Universidad del Magdalena, Santa Marta, Colombia.

Correo electrónico: guillermoceballos@gmail.com

Recibido: 31 de enero del 2014 Aprobado: 4 de abril del 2014

Cómo citar este artículo: Hawkins, S., Valencia, A., Caamaño, B. y Ceballos, C. (2014). Riesgo suicida y desesperanza en pacientes psiquiátricos hospitalizados. Pensando Psicología, 10(17), 43-51. doi: http://dx.doi.org/10.16925/pe.v10i17.783

\begin{abstract}
Resumen
La desesperanza (D) incrementa el riesgo suicida (RS). No se conoce esta información en una muestra representativa de pacientes psiquiátricos hospitalizados en Santa Marta (Colombia). El presente estudio buscó describir la prevalencia y hallar la asociación entre el RS y la $\mathrm{D}$, de acuerdo con variables sociodemográficas y clínicas, en pacientes con trastornos psiquiátricos. Se realizó un estudio transversal, descriptivo correlacional. Se evaluaron 61 pacientes con la Escala de Riesgo Suicida de Plutchik, la Escala de Desesperanza de Beck y un cuestionario de datos sociodemográficos. Como principal resultado, se resalta que el $60,7 \%$ de la muestra presentó RS y el 70,4\% D. Se encontró una correlación significativa entre el RS y la D, también entre el Rs y los intentos previos de suicidio. No se hallaron correlaciones entre el RS, la D y variables sociodemográficas y clínicas. En conclusión, a mayor nivel de D, mayor RS en la población psiquiátrica estudiada.
\end{abstract}

Palabras clave: desesperanza, pacientes psiquiátricos, riesgo suicida, prevalencia.

Suicide Risk and Hopelessness in Hospitalized Psychiatric Patients

\begin{abstract}
Although hopelessness (H) is known to increase the risk of suicide (Rs), this information had not been shown in a representative sample of psychiatric patients hospitalized in Santa Marta (Colombia). This study sought to describe the prevalence of $\mathrm{RS}$ and $\mathrm{H}$, and find a link between them according to socio-demographic and clinical variables, in patients with psychiatric disorders. A cross-sectional, correlational descriptive study was conducted, evaluating 61 patients using the Plutchik Suicide Risk Scale, the Beck Hopelessness Scale, and a socio-demographic data survey. The results highlighted that $60.7 \%$ of the sample presented RS and $70.4 \%$ presented $\mathrm{H}$. A significant correlation was found between $\mathrm{RS}$ and $\mathrm{H}$, and also between RS and previous suicide attempts. No correlation was found between RS, $\mathrm{H}$ and socio-demographic and clinical variables. In conclusion, the higher the level of $\mathrm{H}$, the higher Rs in the study population.
\end{abstract}

Keywords: hopelessness, psychiatric patients, risk of suicide, prevalence.

Risco suicida e desesperança em pacientes psiquiátricos

hospitalizados

\section{Resumo}

A desesperança (D) aumenta o risco suicida (RS). Não se conhece essa informação numa amostra representativa de pacientes psiquiátricos hospitalizados em Santa Marta (Colômbia). O presente estudo pretendeu descrever a prevalência e encontrar a associação entre o RS e a D, de acordo com variáveis sociodemográficas e clínicas, em pacientes com transtornos psiquiátricos. Realizou-se um estudo transversal, descritivo correlacional. Avaliaram-se 61 pacientes com a Escala de Risco Suicida de Plutchik, a Escala de Desesperança de Beck e um questionário de dados sociodemográficos. Como principal resultado, destaca-se que $60,7 \%$ da amostra apresentou Rs e 70,4\%, D. Constatou-se uma correlação significativa entre o Rs e a D, também entre o RS e as prévias tentativas de suicídio. Não se constataram correlações entre o RS, a D e variáveis sociodemográficas e clínicas. Em conclusão, quanto maior nível de D, maior Rs na população psiquiátrica estudada.

Palavras-chave: desesperança, pacientes psiquiátricos, risco suicida, prevalência. 


\section{Introducción $^{1}$}

El suicidio es una problemática significativa de salud pública mundial (Balhara y Verma, 2012; Cañón, 2011; Carvajal y Caro, 2011; Gómez-Durán, Martin-Fumadó y Hurtado-Ruiz, 2012). Según la oms (2012), 1 millón 95 mil personas se suicidan anualmente, lo que equivale al $15,64 \%$ de la población mundial. En los últimos 45 años, las conductas suicidas se han incrementado en un $60 \%$, por lo cual el suicido es la segunda causa de muerte a nivel mundial (Fuentes et al., 2009; CIEs, 2014).

Latinoamérica es uno de los continentes con menor tasa de suicidio; Colombia es el tercer país en el que se reportan mayor cantidad de conductas suicidas, después de Brasil y Cuba. En nuestro país, 1.825 personas al año se quitan la vida, lo que equivale al 3,9\% del total de la población colombiana (CIES, 2014). En el 2010, se presentaron en Santa Marta 18 suicidios consumados, lo que corresponde a una tasa de suicidio del $4,0 \%$ por cada 100000 habitantes de la ciudad (Ceballos y Suárez, 2012).

La conducta suicida se debe a la presencia de trastornos mentales, de los cuales los más frecuentes son tanto el trastorno depresivo mayor y la esquizofrenia (Aliaga, Rodríguez, Ponce, Frisancho y Vereau, 2006; Balhara y Verma, 2012), como otras variables de apoyo social, nivel de estudio y otros parámetros socioeconómicos (Bani-Fatemi et al., 2013; Cañón et al., 2011; Carvajal y Caro, 2011; Labonté et al., 2013; Labonté et al., 2012; oms, 2012; Pavez, Santander, Carranza y Vera, 2009), y se estima que es la principal causa de muerte en pacientes con trastornos mentales en comparación con sujetos sanos de la población general (Capasso, Lineberry, Bostwick, Decker y St Sauveur, 2008; Carlborg, Jokinen, Jonsson, Nordstrom y Nordstrom, 2008; Gómez-Durán, Martin-Fumadó y Hurtado-Ruiz, 2012; Haukka, Suominen, Partonen y Lonnqvist, 2008; Li, Suominen, Partonen y Lonnqvist, 2008; Loas, Azi, Noisette y Yon, 2008; McGirr y Turecki, 2008; Osborn, Levy, Nazareth y King, 2008; Ran et al., 2008; Silverton, Mednick, Holst y John, 2008; Thong, Su, Chan y Chia, 2008; Tidemalm, Langström, Lichtenstein y Runeson, 2008; Zisook et al., 2010), aunque otros estudios reportan que los fallecimientos en población psiquiátrica se relacionan con el padecimiento de enfermedades físicas. Aun así, los resultados de dichos estudios son controvertidos (Tidemalm, Langström, Lichtens-

Los autores declaran que no existe conflicto de intereses. tein y Runeson, 2008; Tiihonen, Lönnqvist y Haukka, 2009; Lawrence, Hancock y Kisely, 2013; Lawrence, Kisely y Pais, 2010; De Hert et al., 2011).

Padecer un trastorno mental reduce la esperanza de vida y aumenta considerablemente el riesgo suicida (Rs) hasta en un $40 \%$ más que el estimado en la población general (Balhara y Verma, 2012; Cañón, 2011; Capasso et al., 2008; Chang et al., 2011; De Hert et al., 2011; Fuentes et al., 2009; Laursen, 2011; López, López y López, 2008; Mojica, Sáenz y Rey-Anacona, 2009; Tidemalm et al., 2008; Tiihonen et al., 2009; Wahlbeck, Westman, Nordentoft, Gissler y Laursen, 2011). El Rs se asocia con diversos factores sociodemográficos y clínicos; diversos estudios han vinculado frecuentemente esta variable con el sexo masculino, tanto en la población general como en la población psiquiátrica (Balhara y Verma, 2012; Hor y Taylor, 2010; López et al., 2008; Ran et al., 2008; Tidemalm et al., 2008), aunque Gómez-Durán et al. (2012) encontraron mayor prevalencia en las pacientes asiáticas con trastornos mentales. Del mismo modo, se ha establecido que el Rs prevalece en la juventud (Cañón, 2011; Gibbons, Brown, Hur, Davis y Mann, 2012; Hor y Taylor, 2010; Osborn et al., 2008; Ran et al., 2008), aunque también se presenta en pacientes de mediana y mayor edad ( $\mathrm{Zi}$ sook et al., 2010). La escolaridad también influye en el rs; en este aspecto, se ha encontrado un mayor riesgo en personas con estudios de educación superior (Hor y Taylor, 2010; McGirr y Turecki, 2008), educación media (López et al., 2008), con estudios primarios o sin ningún nivel educativo (Gómez-Durán et al., 2012). Asimismo, se ha establecido que las personas solteras tienen mayor factor de riesgo para el suicidio en pacientes con trastornos mentales (Acosta et al., 2009; Gómez-Durán et al., 2012; López et al., 2008). Haukka et al. (2008) y Zisook et al. (2010) afirman que entre el $20 \%$ y el $40 \%$ de los pacientes psiquiátricos presentan intentos previos de suicidio, factor que incrementa el Rs (Balhara y Verma, 2012; Gómez-Durán et al., 2012; Haukka et al., 2008; Hor y Taylor, 2010; Li et al., 2008; López et al., 2008; Tidemalm et al., 2008), especialmente cuando se han usado métodos altamente letales (Thong et al., 2008). Otros factores que presentan variación en la asociación respecto al Rs son los antecedentes familiares de enfermedad mental o de suicidio; comorbilidad con enfermedad física crónica o dependencia a sustancias psicoactivas, síntomas positivos y curso de la enfermedad, cantidad de admisiones, contacto con el terapeuta, ingresos obligatorios, fase y adherencia al tratamiento, personalidad, víncu- 
los familiares insatisfactorios, aislamiento social y desempleo. Dichos factores no son independientes sino que muestran asociaciones múltiples entre ellos, por lo que resulta controvertido establecer relaciones directas con el riesgo de suicidio (Balhara y Verma, 2012; Carlborg et al., 2008; Gómez-Durán et al., 2012; Haukka et al., 2008; Hor y Taylor, 2010; Loas et al., 2008; López et al., 2008; McGirr y Turecki, 2008; Silverton et al., 2008; Thong et al., 2008; Zisook et al., 2010).

Una variable mediadora que actúa como predictor del suicidio en los trastornos mentales es la desesperanza (D). Según Stotland (1969), la D consiste en una percepción negativa del futuro inmediato o remoto, que coincide con el tercer componente de la tríada cognitiva propuesto por Beck (citado por Aliaga, Rodríguez, Ponce, Frisancho y Vereau, 2006), el cual presenta características de rasgo predominante (Acosta et al., 2009; Beck, Steer, Kovacs y Garrison, 1985; Beck, Brown, Berchick, Stewart y Steer, 1990). La D es un factor de riesgo para la conducta suicida tanto en la población general como en pacientes psiquiátricos hospitalizados o atendidos ambulatoriamente (Acosta et al., 2009; Aliaga et al., 2006; Beck et al., 1985, 1990; Carvajal y Caro, 2011; Córdova y Rosales, 2010; Gómez-Durán et al., 2012; López et al., 2008; Thong et al., 2008). Aliaga et al. (2006) y Mojica et al. (2009) reportan que comúnmente al menos un $40 \%$ de los pacientes con trastorno mental presentan algún grado leve de $\mathrm{D}$.

$\mathrm{La} \mathrm{D}$, al igual que el Rs, se ha asociado con variables sociodemográficas y clínicas. Aliaga et al. (2006) no hallan correlación con el sexo, pero sí asociación positiva con la edad $(p<0,01)$, aunque esta sea relativamente baja. Adicionalmente se ha encontrado que la $\mathrm{D}$ se asocia con el insight, creencias negativas acerca de la enfermedad, intento de suicidio previo, tratamiento farmacológico y síntomas de la enfermedad (Acosta et al., 2009; Acosta et al., 2013; Hor y Taylor, 2010; Yanos, Roe, Markus y Lysaker, 2008; Zisook et al., 2010). Por otra parte, en un estudio realizado en población sin trastornos mentales, Cañón et al. (2011) encontraron una relación significativa entre la $\mathrm{D}$ y la creencia religiosa.

Frecuentemente se ha asociado la esquizofrenia y la depresión comórbida o como diagnóstico principal a un mayor Rs y D (Aliaga et al., 2006; Balhara y Verma, 2012; Capasso et al., 2008; Carlborg et al., 2008; Gibbons et al., 2012; Gómez-Durán et al., 2012; Haukka et al., 2008; Li et al., 2008; López et al., 2008; McGirr y Turecki, 2008; Mojica et al., 2009; Osborn et al., 2008; Thong et al., 2008; Tidemalm et al., 2008; Zisook et al., 2010). Por último, algunas investigaciones han concluido que el alto índice de suicidio en pacientes con trastornos mentales obedece a la correlación existente entre el Rs y la presencia de D (Cañón et al., 2011; López et al., 2008; Mojica et al., 2009; Scholes y Martin, 2013; Snowden, 2009).

El objetivo general del presente estudio fue determinar la prevalencia y la correlación entre el Rs y la D en una muestra procedente de una población representativa de pacientes con trastornos psiquiátricos hospitalizados en el Hospital Universitario Fernando Troconis de Santa Marta, Colombia, de acuerdo con las variables sociodemográficas y clínicas de edad, sexo, estado civil, religión, lugar de nacimiento, nivel educativo, ocupación, programa adscrito, diagnóstico psiquiátrico, intentos previos de suicidio y antecedentes familiares de suicidio.

\section{Materiales y métodos}

El presente estudio es de corte transversal, con un diseño cuantitativo de tipo descriptivo-correlacional. La muestra estuvo compuesta por 61 pacientes internos con trastornos psiquiátricos del Hospital Universitario Fernando Troconis de Santa Marta, los cuales fueron seleccionados por medio de un muestreo aleatorio simple del total de internos de la institución. La muestra se obtuvo de manera consecutiva durante un mes, teniendo como criterios de exclusión los diagnósticos de retraso mental o trastornos mentales y de comportamiento por patología orgánica. El estudio fue aprobado por la coordinadora del área psiquiátrica y, asimismo, se obtuvo el consentimiento informado de todos los pacientes para su participación en el estudio; en el caso de los pacientes inimputables, el representante legal dio el consentimiento informado. Los psiquiatras a cargo de los pacientes determinaron los diagnósticos a partir de la evaluación en la admisión y el seguimiento, los cuales reposan en las historias clínicas de cada paciente. La aplicación de los instrumentos se llevó a cabo por un único evaluador entrenado durante la estancia hospitalaria de los pacientes, en la fase de estabilización del trastorno. Adicionalmente se aplicó un cuestionario para obtener los datos sociodemográficos; se tuvieron en cuenta datos clínicos como el diagnóstico y el programa al cual estaba adscrito el paciente.

Se aplicó la Escala de Riesgo Suicida de Plutchik (Plutchik, Van Praga, Conte y Picard, 1989) -instrumento de autoaplicación diseñada para identificar a 
los pacientes psiquiátricos suicidas o con Rs- que contiene 15 preguntas con respuestas dicotómicas (sí/no); así, cada afirmación puntúa 1 mientras que la negación puntúa 0 , para un total entre 0 y 15 puntos. No presenta límite de tiempo para su aplicación. En la versión española (Rubio et al., 1998), el corte es de 6 puntos para establecer Rs. Su consistencia interna es de 0,84-0,90 y su fiabilidad test-retest es de 0,89, por lo que es significativamente válida. El onceavo y quinceavo ítems de esta misma escala se usaron para evaluar la conducta suicida previa y los antecedentes familiares de suicidio.

Asimismo se administró la Escala de Desesperanza de Beck -instrumento de autoaplicación en adolescentes y adultos creado para medir el grado de $\mathrm{D}$ en la población con o sin trastornos mentales- que consta de 20 enunciados a los que se responden de forma dicotómica: falso o verdadero. Para establecer la puntuación, en los reactivos 2, 4, 7, 9, 11, 12, 14, 16, 17, 18 y 20 se proporciona 1 punto por cada afirmación y 0 puntos por cada negación; en los reactivos $1,3,5,6,8,10,13$, 15 y 19 se otorga 1 punto por cada negación y 0 puntos por cada afirmación, para un total oscilante entre 0 y 20 puntos clasificables en las categorías de riesgo ausente (0-3), leve (4-8), moderado (9-14) o alto (15-20); por ende, los puntajes mayores a 8 son indicadores de una eventual conducta suicida. El instrumento no tiene límite de tiempo para su aplicación, pero generalmente toma de 5 a 10 minutos. Se recomienda aplicar dicha escala a partir de los 17 años de edad. Su grado de consistencia interna es significativa (Aliaga et al., 2006; Beck et al., 1985; Beck et al., 1990).

El análisis estadístico se realizó mediante el programa spss versión 12.0. Las variables cuantitativas se describieron mediante media aritmética, desviación típica y rango, y las variables cualitativas mediante frecuencias relativas. Las comparaciones entre variables resultado se realizaron utilizando estadísticos no paramétricos. Las asociaciones entre variables demográficas y clínicas continuas, así como en las escalas psicométricas, fueron exploradas mediante coeficiente de correlación de Pearson.

\section{Resultados}

El 65,6\% de los participantes en el estudio era de sexo masculino, y el $34,4 \%$ era de sexo femenino. La edad media reportada fue de 38,52 años, con una desviación típica de 14,001. En cuanto al estado civil, el 63,9\% in- formó ser soltero, el 16,5\% divorciado, el 9,8\% casado, el $4,9 \%$ viudo y en unión libre, respectivamente. En cuanto a la religión, el $44 \%$ manifestaron ser católicos; $20 \%$ evangélicos; $16 \%$ pentecostales y $2 \%$ testigos de Jehová, mientras que el $18 \%$ no pertenecía a ninguna comunidad religiosa.

Asimismo, los participantes provenían de distintos departamentos del país, así: Magdalena 57,3\% (26,2\% son oriundos de Santa Marta), Cesar 9,8\%, Atlántico 6,6\%, Cundinamarca 4,9\%, Bolívar y San Andrés y Providencia 3,3\% respectivamente, Norte de Santander, Santander, Boyacá, Huila, Antioquia, La Guajira, Chocó, Tolima y Quindío 1,6\% respectivamente.

En cuanto al nivel educativo, el $24,6 \%$ tuvo acceso a estudios profesionales y técnicos, el $34,4 \%$ culminaron el nivel de educación media mientras que el 18\% no, y el $23 \%$ realizaron estudios de básica primaria (de estos, el $24,6 \%$ no los culminaron o nunca tuvieron acceso a ellos).

Con respecto a la ocupación de los participantes, el $16,4 \%$ se encontraban desempleados; la ocupación prevalente fue comerciante $(14,8 \%)$, seguida de ama de casa $(13,1 \%)$ y el $55,7 \%$ restante eran meseros, carpinteros, empleados domésticos, conductores, de oficios varios, pescadores, albañiles, mecánicos, fumigadores, herreros, de seguridad, campesinos y estudiantes.

El 63,9\% de los pacientes se encontraban adscritos a los programas de Hospitalización general, el 29,5\% Inimputables y el 6,6\% Hospital día.

El diagnóstico psiquiátrico prevalente fue la esquizofrenia con $50,8 \%$, seguido por $16,4 \%$ de trastornos psicóticos agudos y transitorios, $13,1 \%$ de trastornos esquizoafectivos, $4,9 \%$ de trastorno mental y de comportamiento debido al consumo de drogas y otros psicotrópicos, $4,9 \%$ de bipolar afectivo y depresivo recurrente, $3,3 \%$ de episodios depresivos, y 1,6\% de trastornos de personalidad. El 32,8\% de los pacientes ha presentado un intento de suicidio.

En cuanto al historial de suicidio en la familia, el $14,8 \%$ cuenta con este antecedente. El $60,7 \%$ de la población psiquiátrica encuestada presenta rs, mientras que el 39,3\% no presenta dicho riesgo (tabla 1 ).

Como se observa en la tabla 2, el 70,4\% de los participantes presenta algún grado de $\mathrm{D}$ (45,5\% leve, $18 \%$ moderada, $6,6 \%$ severa). No se encontró diferencia estadísticamente significativa.

Asimismo, se ha establecido la prevalencia de las variables sociodemográficas en la población que presenta algún nivel de D: el 75\% de los hombres están desesperanzados, mientras que el $61,9 \%$ de las mujeres 
Tabla 1

Nivel de riesgo suicida (RS)

\begin{tabular}{lcccc}
\hline \multicolumn{1}{c}{ Válidos } & Frecuencia & Porcentaje (\%) & Porcentaje válido (\%) & Porcentaje acumulado (\%) \\
\hline Con riesgo suicida & 37 & 60,7 & 60,7 & 60,7 \\
Sin riesgo suicida & 24 & 39,3 & 39,3 & 100,0 \\
Total & 61 & 100,0 & 100,0 & \\
\hline
\end{tabular}

Nota. Elaboración propia.

Tabla 2

Nivel de desesperanza (D)

\begin{tabular}{lcccc}
\hline \multicolumn{1}{c}{ Válidos } & Frecuencia & Porcentaje (\%) & Porcentaje válido (\%) & Porcentaje acumulado (\%) \\
\hline Asintomático & 18 & 29,5 & 29,5 & 29,5 \\
Leve & 28 & 45,9 & 45,9 & 75,4 \\
Moderado & 11 & 18,0 & 18,0 & 93,4 \\
Severo & 4 & 6,6 & 6,6 & 100,0 \\
Total & 61 & 100,0 & 100,0 & \\
\hline
\end{tabular}

Nota. Elaboración propia.

Tabla 3

Riesgo suicida por variable sociodemográfica

\begin{tabular}{lcc}
\hline \multicolumn{1}{c}{ Variable } & Sí Rs (\%) & No Rs (\%) \\
\hline Sexo masculino & 60,0 & 40,0 \\
Sexo femenino & 61,9 & 38,1 \\
Edad (15-34 años) & 62,5 & 37,5 \\
Edad (35 o más años) & 58,5 & 41,5 \\
Diagnóstico de esquizofrenia & 48,4 & 51,6 \\
Pacientes hospitalizados & 69,2 & 30,8 \\
Pacientes inimputables & 38,9 & 61,1 \\
Estado civil soltero & 64,1 & 35,9 \\
Sin religión & 75,0 & 25,0 \\
Con alguna religión & 58,5 & 41,5 \\
Sin nivel educativo & 80,0 & 20,0 \\
Nivel educativo de primaria & 65,0 & 35,0 \\
Nivel de educación media & 47,0 & 53,0 \\
Total & $37(60,7)$ & $24(39,3)$ \\
\hline
\end{tabular}

Nota. Elaboración propia.

lo están. El 75\% de los pacientes entre 15 y 34 años presentan $\mathrm{D}$, mientras que de la población mayor de 34 años sólo el 66,7\% la presenta. En cuanto al diagnóstico de los pacientes, se encontró que de las personas con esquizofrenia un $67,7 \%$ está desesperanzadas. En lo referente al programa al que pertenecen, se halló que el $71,8 \%$ de los que se encuentran en hospitalización general presentan $\mathrm{D}$, mientras que de los pacientes inimputables sólo el $61,1 \%$ la presenta. En cuanto al estado civil, un $71,8 \%$ de las personas solteras presentan D. En lo referente a la religión, el 75,6\% de las personas que no practican religión alguna presentan $\mathrm{D}$, mientras que el $69,8 \%$ de quienes pertenecen a un grupo religioso la presentan. Respecto al nivel de educación, el 100\% de las personas que no alcanzaron ningún nivel educativo presentan $\mathrm{D}$, mientras que de las personas que realizaron estudios primarios el $75 \%$ la presentan y de las personas con educación media sólo el 52,47\%. 
Se encontró una correlación significativa entre el Rs y los intentos previos de suicidio según el coeficiente de correlación de Pearson, con una significancia bilateral de 0 (tabla 3). No se hallaron correlaciones significativas entre el Rs ni la D con variables sociodemográficas como edad, estado civil, sexo, nivel de educación, grupo religioso y ocupación.

Se encontró correlación significativa entre las variables RS y D, según el coeficiente de correlación de Pearson, con una significancia bilateral de 0,000 (tablas 4 y 5 ).

Tabla 4

Correlación entre riesgo suicida e intentos suicidas previos

\begin{tabular}{llcc}
\hline & & Nivel de riesgo suicida & $\begin{array}{c}\text { ¿Alguna vez ha intentado } \\
\text { quitarse la vida? }\end{array}$ \\
\hline Nivel de riesgo suicida & Correlación de Pearson & 1 & $0,563^{* *}$ \\
& Sig. (bilateral) & & 0,000 \\
& $\mathrm{~N}$ & 61 & 61 \\
\hline \multirow{2}{*}{ Ha intentado alguna vez quitarse la vida? } & Correlación de Pearson & $0,563^{* *}$ & 1 \\
& Sig. (bilateral) & 0,000 & 61 \\
\hline
\end{tabular}

Nota. Elaboración propia.

${ }^{* *}$ La correlación es significativa al nivel 0,01 (bilateral)

Tabla 5

Correlación entre riesgo suicida y desesperanza

\begin{tabular}{llll}
\hline & & Nivel de desesperanza & Nivel de riesgo suicida \\
\hline Nivel de desesperanza & Correlación de Pearson & 1 & $-0,562^{* *}$ \\
& Sig. (bilateral) & & 0,000 \\
& $\mathrm{~N}$ & 61 & 61 \\
\hline Nivel de riesgo suicida & Correlación de Pearson & $-0,562^{* *}$ & 1 \\
& Sig. (bilateral) & 0,000 & 61 \\
\hline
\end{tabular}

Nota. Elaboración propia.

** La correlación es significativa al nivel 0,01 (bilateral)

\section{Discusión}

La presente investigación muestra que el $60,7 \%$ de la población psiquiátrica estudiada presenta Rs. Este dato es consistente con los hallazgos de López et al. (2008), y es más elevada que la reportada en pacientes con esquizofrenia (Acosta et al., 2009; Balhara y Verma, 2012; Bani-Fatemi et al., 2013), trastorno esquizoafectivo (Zisook et al., 2010) e inimputables (Mojica et al., 2009). Este riesgo se asoció significativamente con intentos de suicidio previos (Balhara y Verma, 2012; Gómez-Durán et al., 2012; Haukka et al., 2008; Hor y Taylor, 2010; Li et al., 2008; López et al., 2008; Pavez, Santander, Carranza y Vera-Villaroel, 2009; Tidemalm et al., 2008), por lo que los intentos de suicidio previos se asociarían con mayor riesgo de suicidio en esta población, independientemente de su proximidad en el tiempo al momento de la inclusión en el estudio. No se hallaron correlaciones significativas con las variables sociodemográficas y clínicas como sexo, edad, estado civil, grupo religioso, nivel de educación, ocupación, programa adscrito, diagnóstico y antecedente familiar de suicidio. Algunos de los resultados de prevalencia del Rs concuerdan con los datos reportados por diversas investigaciones, hallándose que la mayoría de los pacientes son solteros (Acosta et al., 2009; Gómez-Durán et al., 2012; López et al., 2008), jóvenes y adultos tempranos (Cañón, 2011; Gibbons et al., 2012; Hor y Taylor, 2010; Osborn et al., 2008; Ran et al., 2008) y presentan esquizofrenia (Acosta et al., 2013; Balhara y Verma, 2012; Capasso et al., 2008; Carlborg et al., 2008; Gómez-Durán et al., 2012; McGirr y Turecki, 2008; Mojica et al., 2009; Silverton et al., 2008; Thong et al., 2008; Tidemalm et al., 2008; Zisook et al., 2010), sin algún nivel educativo o con estudios primarios (GómezDurán et al., 2012). 
Asimismo, la presente investigación halló que el $70,4 \%$ de los pacientes con trastornos psiquiátricos presenta algún grado de $\mathrm{D}(45,5 \%$ leve, $18,03 \%$ moderada, $6,55 \%$ severa), cifra mayor a la reportada por Aliaga et al. (2006) y Mojica et al. (2009), apoyando la hipótesis de que este factor es una variable mediadora que actúa como predictor del suicidio en los trastornos mentales (Acosta et al., 2009), que también suelen presentar niveles de D asintomático (49\%) y leve (40,2\%), pero no es común que presenten D moderada $(8,8 \%)$ y severa (2\%; Aliaga et al., 2006). Tampoco se encontraron correlaciones significativas con las variables sociodemográficas y clínicas como sexo (Aliaga et al., 2006), estado civil, nivel de educación, ocupación, programa adscrito, diagnóstico, intento de suicidio previo ni antecedente familiar de suicidio (Acosta et al., 2009; Mojica et al., 2009). Se hallan resultados contradictorios respecto a la edad, que resultó ser directamente proporcional a la $\mathrm{D}$, aunque con un nivel de significancia bajo (Aliaga et al., 2006) y el grupo religioso asociado significativamente con menores niveles de D en población sin trastornos mentales (Cañón et al., 2011). Asimismo, respecto a la prevalencia, se encontró que la mayoría de los pacientes con esquizofrenia presentan D (Aliaga et al., 2006; Mojica et al., 2009). No se detectaron diferencias de sexo estadísticamente significativas en cuanto a la prevalencia de Rs y $\mathrm{D}$, lo cual indica que los pacientes con trastornos mentales se encuentran en riesgo de cometer un acto suicida (Carlborg et al., 2008). Se sugiere mayor atención investigativa y asistencial en la población femenina respecto al Rs.

Por último, la correlación significativa entre la $\mathrm{D}$ y el rs coincide con constructo de la D como variable mediadora que incrementa el Rs y contribuye al alto índice de suicidio en pacientes con trastornos mentales. Esto sería concordante con los hallazgos de Acosta et al. (2009); Cañón et al. (2011); López et al. (2008); Mojica et al. (2009); Scholes y Martin (2013) y Snowden (2009).

Los antecedentes consultados para la presente investigación indican que los pacientes psiquiátricos presentan una tasa de mortalidad por suicidio más alta que la población general, por lo que resulta importante realizar una caracterización de los pacientes con trastornos mentales que presentan Rs y la D a partir de la evaluación de estos factores durante la instancia hospitalaria, con el fin de identificar factores de riesgo, promover la atención y vigilancia continuas, e incluso después del alta; también se debe tener en cuenta el desarrollo de intervenciones para la prevención del suicidio en esta población, especialmente centrada en la D para atender esta problemática significativa de salud pública.
Es importante aclarar las limitaciones que presentó este estudio. En primer lugar, el tamaño de las muestras de algunos grupos diagnósticos es relativamente pequeño, de manera que no alcanza a ser significativa para el hallazgo de su prevalencia en cuanto al Rs ni a la $\mathrm{D}$, por lo que se requieren más investigaciones al respecto que controlen esta falencia en esta investigación. En segundo lugar, el diseño transversal usado en el estudio no permite establecer relaciones causa-efecto en la interpretación de los resultados, por lo que sólo se pueden formular hipótesis al respecto. A pesar de ello, el diseño del presente estudio permite detectar medidas de asociación y no predictores de suicidio.

\section{Conclusión}

El suicidio es una problemática importante de salud pública a nivel internacional que se ha incrementado con el transcurso de los años. La conducta suicida se debe a la interacción de factores biopsicosociales. Padecer un trastorno mental aumenta el Rs hasta en un $40 \%$ en comparación con la población general. La D es una variable mediadora que acrecienta el Rs en la población psiquiátrica, constructo sustentado por la correlación significativa entre ambas variables. El RS y la D son prevalentes en esta población $(60,7 \%$ y $70,4 \%$ respectivamente), además de que se encuentra una fuerte asociación entre altos niveles de D y mayor Rs. Tradicionalmente, el Rs y la $\mathrm{D}$ se han asociado con diversos factores sociodemográficos y clínicos, pero no se hallaron correlaciones significativas al respecto, excepto entre el intento previo de suicidio y el Rs, por lo que podría establecerse como factor de riesgo en esta población independientemente de su proximidad en el tiempo.

Se requieren más investigaciones con diseño longitudinal que permitan una mayor consistencia y potencia en el establecimiento de relaciones causa-efecto en la interpretación de los resultados, además de estudios en muestras significativas de pacientes de diversas categorías diagnósticas de trastornos mentales que permitan establecer la prevalencia del Rs y la D en cada una. Se recomienda realizar una caracterización de los pacientes con trastornos mentales que presentan Rs y D para identificar factores de riesgo en esta población, incluir estas escalas en la evaluación integral de los pacientes durante su instancia hospitalaria, y además promover la atención y prevención continuas ante el suicidio para atender esta problemática de salud pública internacional. 


\section{Referencias}

Acosta, F., Aguilar, E., Cejas, M., Gracia, R., Caballero, A. y Siris, S. (2009). A Prospective Study of the Psychopathological Variables Associated with Suicidality among Schizophrenic Patients. Actas Españolas de Psiquiatría, 37(1), 42-48.

Acosta, F., Aguilar, E., Cejas, M. y Gracia, R. (2013). Beliefs about Illness and their Relationship with Hopelessness, Depression, Insight and Suicide Attempts in Schizophrenia. Psychiatria Danubina, 25(1), 49-54. Recuperado de http://www.hdbp.org/psychiatria_danubina/pdf/ dnb_vol25_no1/dnb_vol25_no1_49.pdf

Aliaga, J., Rodríguez, L., Ponce, C., Frisancho A. y Vereau J. (2006). Escala de Desesperanza de Beck (bhs): Adaptación y características psicométricas. Revista de Investigación en Psicología, 9(1), 69-79.

Balhara, Y. y Verma, R. (2012). Schizophrenia and Suicide. East Asian Archives of Psychiatry, 22(3), 126-133.

Bani-Fatemi, A., Gonçalves, V., Zai, C., De Souza, R., Le Foll, B., Kennedy, J. L; Wong, A. H y De Luca, V. (2013). Analysis of cpg snps in 34 Genes: Association Test with Suicide Attempt in Schizophrenia. Schizophrenia Research, 147, 262-268.

Beck, A., Steer R., Kovacs, M. y Garrison, B. (1985). Hopelessness and Eventual Suicide: A 10-year Prospective Study of Patients Hospitalized with Suicidal Ideation. American Journal Psychiatry, 142(5), 559-563.

Beck A., Brown G., Berchick R., Stewart B. y Steer, R. (1990). The Relationship between Hopelessness and Ultimate Suicide: A Replication with Psychiatric Outpatients. American Journal Psychiatry, 147(2), 190-195.

Cañón, S. (2011). Factores de riesgo asociados a conductas suicidas en niños y adolescentes. Archivos de Medicina, 11(1), 62-67.

Cañón, S., Castaño, J., Atehortúa, B., Botero, P., García, L., Rodríguez, L. M.; Tovar, C. A. y Rincón, E. (2011). Factor de riesgo para suicidio según dos cuestionarios y factores asociados en población estudiantil de la Universidad de Manizales (Colombia). Psicología desde el Caribe, 29(3), 632-664.

Capasso, R., Lineberry, T., Bostwick, J., Decker, P. y St Sauveur, J. (2008). Mortality in Schizophrenia and Schizoaffective Disorder: An Olmsted County, Minnesota Cohort: 19502005. Schizophrenia Research, 98(1-3), 287-294.

Carlborg, A., Jokinen, J., Jonsson, E., Nordstrom, A. y Nordstrom, P. (2008). Long-Term Suicide Risk in Schizophrenia Spectrum Psychoses: Survival Analysis by Gender. Archives of Suicide Research, 12(4), 347-351.

Carvajal, G. y Caro, C. (2011). Ideación suicida en la adolescencia: Una explicación desde tres de sus variables asociadas en Bogotá. Colombia Médica, 42(2-1), 45-56.

Ceballos, G. y Suárez, Y. (2012). Epidemiología del suicidio en el Distrito T. C. e H. de Santa Marta y en el departamento del Magdalena años 2004-2011. En G. Ceballos y Y. Suárez (Eds.), El suicidio (pp. 23-32). Santa Marta: Editorial Unimagdalena.

Chang, C., Hayes, R., Perera, G., Broadbent, M., Fernandes, A., Lee, W. E; Hotopf, M, y Stewart, R. (2011). Life Expectancy at Birth for People with Serious Mental Illness and Other Major Disorders from a Secondary Mental Health Care Case Register in London. PlosOne, 6(5), 1-6. doi: 10.1371/journal.pone.0019590

Córdova, M. y Rosales, J. (2010). La desesperanza de riesgo en jóvenes mexicanos y aspectos sociodemográficos asociados: Diferencias por sexo. Psicología y Salud, 20(2), 195-201.

Corporación Iberoamericana de Estudios (CIEs) - Fundación Santa Fe de Bogotá. (2014). Seguimiento al sector salud en Colombia. Así Vamos en Salud: Indicadores. Estado de salud. Tasa de suicidio. Bogotá: Así Vamos en Salud. Recuperado de http://www.asivamosensalud.org/inidicadores/estado-de-salud/grafica.ver/56

De Hert, M., Correll, C., Bobes, J., Cetkovich-Bakmas, M., Cohen, D., Asai, I., Detraux, J., Gautam, S., Möller, H. J., Ndetei, D. M., Newcomer, J. W., Uwakwe, R. y Leucht, S. (2011). Physical illness in patients with severe mental disorders. I. prevalence, impact of medications and disparities in health care. World Psychiatry, 10(1), 52-77. doi: 10.1002/j.2051-5545.2011.tb00014.x

Fuentes, M., González, A., Castaño, J., Hurtado, C., Ocampo, P., Páez, M. y Zuluaga, L. (2009). Riesgo suicida y factores relacionados en estudiantes de $6^{\circ}$ a $11^{\circ}$ grado en colegios de la ciudad de Manizales (Colombia), 20072008. Archivos de Medicina, 9(2), 110-122.

Gibbons, R., Brown, C., Hur, K., Davis, J. y Mann, J. (2012). Suicidal thoughts and behavior with antidepressant treatment: reanalysis of the randomized placebo-controlled studies of fluoxetine and venlafaxine. Archives of General Psychiatry, 69(6), 580-587. doi:10.1001/archgenpsychiatry.2011.2048

Gómez-Durán, E., Martín-Fumadó, C. y Hurtado-Ruiz, G. (2012). Clinical and Epidemiological Aspects of Suicide in Patients with Schizophrenia. Actas Españolas de Psiquiatría, 40(6), 333-445.

Haukka, J., Suominen, K., Partonen, T. y Lonnqvist, J. (2008). Determinants and outcomes of serious attempted suicide: A nationwide study in Finland, 1996-2003. American Journal Epidemiology, 167(10), 155-163.

Hor, K. y Taylor, M. (2010). Suicide and Schizophrenia: a Systematic Review of Rates and Risk Factors. Journal of Psychopharmacology, 24(4), 81-90. doi: $10.1177 / 1359786810385490$.

Labonté, B., Yerko, V., Gross, J., Mechawar, N., Meaney, M., Szyf, M. y Turecki, G. (2012). Differential Glucocorticoid Receptor Exon 1(B), 1(C), and 1(H) Expression 
and Methylation in Suicide Completers with a History of Childhood Abuse. Biological Psychiatry, 72(1), 41-48.

Labonté, B., Suderman, M., Maussion, G., Lopez, J., NavarroSánchez, L., Yerko, V., Mechawar, N.; Szyf, M.; Meaney, M. J. y Turecki, G. (2013). Genome-Wide Methylation Changes in the Brains of Suicide Completers. American Journal Psychiatry, 170(5), 511-520.

Laursen, T. (2011). Life expectancy among persons with schizophrenia or bipolar affective disorder. Schizophrenia Research, 131(1-3), 101-104.

Lawrence, D., Hancock, K. y Kisely, S. (2013). The Gap in Life Expectancy from Preventable Physical Illness in Psychiatric Patients in Western Australia: Retrospective Analysis of Population Based Registers. British Medical Journal, 346(539), 1-14.

Lawrence, D., Kisely, S. y Pais, J. (2010). The Epidemiology of Excess Mortality in People with Mental Illness. Canadian Journal of Psychiatry, 55(12), 752-760.

Li, J., Ran, M., Hao, Y., Zhao, Z., Guo, Y., Su, J. y Lu, H. (2008). Inpatient Suicide in a Chinese Psychiatric Hospital [versión electrónica]. Suicide and Life- Threatening Behavior, 38(4), 449-55. Recuperado de PubMed Database/ 18724793.

Loas, G., Azi, A., Noisette, C. y Yon, V. (2008). Mortalité et causes de décès dans la schizophrénie: étude prospective entre dix et 14 ans d'une cohorte de 150 sujets. L`Encéphale, 34(1), 54-60.

López, F., López, F. y López, S. (2008). Ideación suicida y desesperanza en pacientes psiquiátricos hospitalizados. Alcmeon, Revista Argentina de Clínica Neuropsiquiátrica, 14(3), 33-41.

McGirr, A. y Turecki, G. (2008). What is Specific to Suicide in Schizophrenia Disorder? Demographic, Clinical and Behavioural Dimensions. Schizophrenia. Research, 98(1-3), 217-224.

Mojica, C., Sáenz, D. y Rey-Anacona, C. (2009). Riesgo suicida, desesperanza y depresión en internos de un establecimiento carcelario colombiano. Revista Colombiana de Psiquiatría, 38(4), 681-692.

Organización Mundial de la Salud (oms). (2012). Salud mental: prevención del suicidio (supre). Ginebra: OMs. Recuperado de http://www.who.int/es/

Osborn, D., Levy, G., Nazareth, I. y King, M. (2008). Suicide and Severe Mental Illnesses. Cohort Study Within the UK General Practice Research Database. Schizophrenia Research, 99(1-3), 134-138.

Pavez, P., Santander, N., Carranza, J. y Vera-Villaroel, P. (2009). Factores de riesgo familiares asociados a la conducta suicida en adolescentes con trastorno depresivo. Revista Médica de Chile, 137(2), 226-233.

Plutchik, R., Van Praga, H. M., Conte, H. R. y Picard, S. (1989). Correlates of Suicide and Violence Risk: The Suicide Risk Measure. Comprehensive Psychiatry, 30(4), 296-302.
Ran, M., Chan, C., Chen, E., Tang, C., Lin, F., Li, L. y Conwell, Y. (2008). Mortality of Geriatric and Younger Patients with Schizophrenia in the Community. Suicide and LifeThreatening Behavior, 38(2), 143-151.

Rubio, G., Montero, I., Jáuregui, J., Villanueva, R., Casado, M. A., Marín, J. J. y Santo Domingo, J. (1998). Validación de la escala de riesgo suicida de Plutchik en población española. Arch Neurobiol, 61(2), 143-152.

Scholes, B. y Martin, C. (2013). Measuring depression in schizophrenia with questionnaires. Journal of Psychiatric and Mental Health Nursing, 20(1), 17-22. doi: 10.1111/j.1365-2850.2012.01877.x

Silverton, L., Mednick, S., Holst, C. y John, R. (2008). High Social Class and Suicide in Persons at Risk for Schizophrenia. Acta Psychiatrica Scandinavica, 117(3), 192-197.

Snowden, A. (2009). Classification of Schizophrenia. Part One: the Enduring Existence of Madness. British Journal of Nursing, 18(19), 1176-1180.

Stotland, E. (1969). Exploratory Investigations of Empathy. In L. Berkowitz (Ed.), Advances in Experimental Social Psychology (Vol. 4, pp. 271-313). New York: Academic Press.

Thong, J., Su, A., Chan, Y. y Chia, B. (2008). Suicide in Psychiatric Patients: Case-Control Study in Singapore. Australian and New Zealand Journal of Psychiatry, 42(6), 509-519.

Tidemalm, D., Langström, N., Lichtenstein, P. y Runeson, B. (2008). Risk of Suicide after Suicide Attempt according to Coexisting Psychiatric Disorder: Swedish Cohort Study with Long Term Follow-Up. British Medical Journal, 337(7682), 1-6.

Tiihonen, J., Lönnqvist, J. y Haukka, J. (2009). Correlacionan el uso de antipsicóticos con los índices de mortalidad en individuos con esquizofrenia. The Lancet, 374(9690), 620-627. Recuperado de http://www.intramed.net/contenidover.asp? contenidoID $=65351$

Wahlbeck, K., Westman, J., Nordentoft, M., Gissler, M. y Laursen, T. (2011). Outcomes of Nordic Mental Health Systems: Life Expectancy of Patients with Mental Disorders. British Journal of Psychiatry, 131(1-3), 101-104. doi: 10.1192/bjp.bp.110.085100

Yanos, P., Roe, D., Markus, K. y Lysaker, P. (2008). Pathways between internalized stigma and outcomes related to recovery in schizophrenia spectrum disorders. Psychiatric Services, 59(12), 1437-1442.

Zisook, S., Kasckow, J., Lanouette, N., Golshan, S., Fellows, I., Vahia, I.; Mohamed, S. y Rao, S. (2010). Augmentation with Citalopram for Suicidal Ideation in Middle-Aged and Older Outpatients with Schizophrenia and Schizoaffective Disorder who Have Subthreshold Depressive Symptoms: A Randomized Controlled Trial. Journal of Clinical Psychiatry, 71(7), 915-922. 